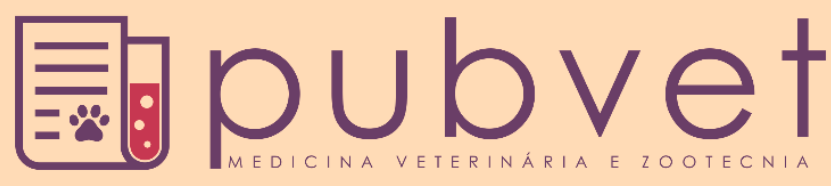

https://doi.org/10.31533/pubvet.v15n06a840.1-5

\title{
Cicatriz tóxica de cesariana em cadela tratada com Terapia Neural
}

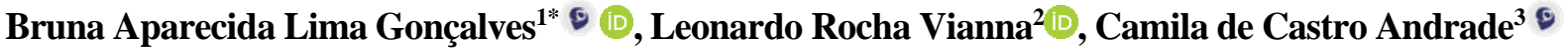 \\ ${ }^{I}$ Mestre em Ciência Animal pela Universidade Federal de Minas Gerais. Acupunturista, Terapeuta Neural. Brasil \\ ${ }^{2}$ Mestre em Clínica e Cirurgia Veterinária pela Universidade Federal de Minas Gerais. Homeopata, Acupunturista, Medicina Tradicional \\ Chinesa, Terapeuta Neural. \\ ${ }^{3}$ Graduação Medicina Veterinária pela Pontifícia Universidade Católica de Minas Gerais Betim-MG Brasil. E programa de aprimoramento \\ profissional em anestesiologia, clínica médica e cirúrgica pela PUC Minas Betim. \\ *Autor para correspondência, E-mail: bruna_alg@yahoo.com.br.
}

Resumo. A Terapia Neural consiste em uma terapia reguladora que busca ativar os mecanismos autorreguladores do organismo, por meio da utilização de anestésicos locais em baixas concentrações, exemplo, o cloridrato de procaína, em determinadas regiões corporais, como pontos de acupuntura, cicatrizes, articulações, de forma venosa entre outras. O objetivo da Terapia Neural é dar um impulso neural terapêutico para ativar o organismo ante um processo de autocura, atuando na regulação e equilíbrio do sistema nervoso autônomo. As áreas de aplicação são escolhidas por meio do histórico de vida do paciente. Essas áreas podem ser campos de interferência, regiões irritativas ao sistema nervoso que geram memórias nocivas ao organismo. Esse trabalho objetiva demonstrar a eficácia da Terapia Neural em uma cadela que não ciclava e após a aplicação de cloridrato de procaína a $0,7 \%$ na cicatriz interferente de cesariana ela voltou a ter cio.

Palavras chaves: Anestro, cio, campo interferente, infertilidade, procaína

\section{Toxic cesarean scar in female dog treated with Neural Therapy}

\begin{abstract}
Neural Therapy consists of a regulatory therapy that seeks to activate the body's self-regulating mechanisms, through the use of local anesthetics in low concentrations, such as procaine hydrochloride, in certain body regions, such as acupuncture points, scars, joints, so among others. The goal of Neural Therapy is to give a therapeutic neural impulse to activate the organism for the self-healing process, acting on the regulation and balance of the autonomic nervous system. The application areas are chosen through the patient's life history. These areas can be fields of interference, irritating regions to the nervous system that generate harmful memories to the organism. This work aims to demonstrate the effectiveness of Neural Therapy in a dog that did not cycle and after the application of $0.7 \%$ procaine hydrochloride in the interfering scar of cesarean section she returned to heat.
\end{abstract}

Keywords: Anestro, estrus, interfering field, infertility, procaine

\section{Cicatriz de cesárea tóxica en perra tratada con Terapia Neural}

Resumen. La Terapia Neural consiste en una terapia reguladora que busca activar los mecanismos de autorregulación del organismo, mediante el uso de anestésicos locales en bajas concentraciones, ejemplo, el clorhidrato de procaína, en determinadas regiones corporales, como puntos de acupuntura, cicatrices, articulaciones, etc. entre otros. El objetivo de la Terapia Neural es dar un impulso neuronal terapéutico para activar el organismo hacia el proceso de autocuración, actuando sobre la regulación y equilibrio del sistema nervioso autónomo. Las áreas de aplicación se eligen a través de la historia de vida del paciente. Estas áreas pueden ser campos de interferencia, regiones irritantes del sistema 
nervioso que generan memorias dañinas al organismo. Este trabajo tiene como objetivo demostrar la efectividad de la Terapia Neural en una perra que no cicló y tras la aplicación de hidrocloruro de procaína al $0,7 \%$ en la cicatriz interferente de la cesárea volvió a presentar celo.

Palabras claves: Anestro, estro, campo interferente, infertilidad, procaína

\section{Introdução}

A Terapia Neural (TN) se iniciou na Rússia, Alemanha e Inglaterra em meados do século XIX a partir de observações dos russos, Speransky, Vischevsky e Bikov, na Alemanha por Spiess, Leriche e Schleich e na Inglaterra por Head. Nas primeiras décadas do século XX dois médicos alemães se destacaram Ferdinand e Walter Huneke e aprofundaram os estudos sobre a Terapia Neural (Cruz \& Naffah, 2011). Esta atua no Sistema Nervoso que regula e comanda todas as reações do organismo, incluindo a sua participação em todos os processos mentais e emocionais (Vianna \& Gonçalves, 2017). Está difundida na Alemanha, Áustria, Argentina, Colômbia, Costa Rica, Cuba, Chile, Equador, Espanha, México, Rússia, Suíça, Uruguai (Vianna \& Gonçalves, 2016).

A Terapia Neural é uma modalidade de tratamento que utiliza a aplicação de anestésicos locais em baixas concentrações, pode ser utilizada em desordens funcionais, doenças inflamatórias, dores agudas e crônicas. É um tratamento autorregulatório (Egli et al., 2015). Utiliza a aplicação de anestésicos locais como lidocaína e procaína em concentrações que variam de 0,1 a $1 \%$ estes possuem propriedades dielétricas e com isso promovem o restabelecimento do potencial fisiológico de membrana celular (Cruz \& Naffah, 2011).

As células normais possuem uma carga elétrica que varia de +-40 a +-90 mV alterações nas cargas destas células em qualquer local do corpo podem provocar transtornos que desenvolvem em enfermidades (Álvarez-Edwin et al. 2017). A célula enferma perde a capacidade de responder a estímulos e permanece despolarizada, mas pode recuperar a função fisiológica normal após um estímulo elétrico externo ou o da substância neural terapêutica que possibilita a repolarização da membrana da célula (Cruz \& Naffah, 2011). O cloridrato de procaína a 0,7\% com bicabornato de sódio apresenta um potencial elétrico de até $207 \mathrm{mV}$ (Vianna \& Goçalves, 2020).

As áreas corporais cronicamente alteradas causam danos e interferências locais e/ou gerais e são denominadas na Terapia Neural de "campos interferentes". Um campo de interferência é uma área irritada que pode causar patologias. Após o estímulo neural terapêutico essa área se restabelece e volta à normalidade. Uma cicatriz pode ser um campo interferente, por exemplo (Bella, 2011). Pischinger observou a normalização da resistência elétrica após a aplicação de anestésicos locais em cicatrizes que antes apresentavam resistências elétricas elevadas, e o desaparecimento dos sintomas clínicos imediato em alguns casos (Gonçalves et al., 2019).

Segundo Bella (2011) em humanos as cicatrizes, as amigdalas e os dentes são fortes campos de interferência. As cicatrizes interferentes possuem uma alta e anormal resistência em relação a pele normal, uma resistência até 10 vezes maior. Uma cicatriz que não se recompõe adequadamente pode se tornar um campo interferente.

Uma cicatriz que atravessa um meridiano de acupuntura é mais provável de causar distúrbios nas estruturas que estão relacionadas a este meridiano do que em qualquer outro lugar. Para exemplo, uma cicatriz da vesícula biliar muitas vezes é responsável por dor crônica no quadril (Bella, 2011; Klinghardt. $\underline{1993}$.

A procaína e seus derivados também têm sido estudados como agentes antitumorais agindo pela inibição da DNA metil transferase e inibindo o crescimento de células cancerígenas. Vários trabalhos têm enfatizado a procaína como um agente para terapia anticâncer baseada na sua ação epigenética (Gonçalves et al., 2020b; Muri et al., 2010). Além das suas propriedades anti-inflamatória e antimicrobiana (Cassuto et al., 2006; Gonçalves et al., 2020a). A procaína também possui ação: antiadrenérgica, anti-histamínica, vasodilatadora, analgésica e capilarizante (Bella, 2011).

O primeiro anestésico local descoberto foi a cocaína no início do século XIX. A cocaína foi primeiramente isolada em 1860 por Albert Niemann. Depois Sigmund Freud estudou suas ações 
fisiológicas e Carl Koller introduziu a cocaína na prática clínica, em 1884, como um anestésico de uso tópico em cirurgias oftalmológicas. Devido a sua toxicidade e propriedades aditivas, iniciou-se a pesquisa para a descoberta de substituintes sintéticos da cocaína, resultando na síntese da procaína, a qual se tornou o protótipo para os anestésicos locais por aproximadamente meio século. E somente em 1943 que a lidocaína foi sintetizada originando, assim, a classe das amino amidas (Muri et al., 2010). A procaína foi sintetizada por Alfred Einhorn em 1905 (Akcal et al., 2015).

Em 1906 Spiess observa após aplicação regional de procaína a melhora de feridas, Cathelin realiza injeção epidural de cocaína, Leriche em 1924 realiza anestesia do gânglio estrelado com procaína, curando a síndrome de horner; Ferdinand Huneke em 1926 aplica acidentalmente a procaína intravenosa para tratamento de enxaqueca "intratável" e obtém êxito (Bella, 2011; Klinghardt, 1993).

A Terapia Neural pode ser utilizada em inúmeras enfermidades como transtornos reumáticos, neurológicos, ginecológicos, enxaquecas, amigdalites, sinusites, otites, asma, afecções dermatológicas dentre outras (De la Torres Rosés \& Mendoza, 2008). Segundo Weinschenk (2012) a Terapia Neural pode ser utilizada em esclerose múltipla, colite, pancreatite, tinido, vulvodina, enxaqueca, neuralgia pós herpética, dor visceral, dor miofascial, síndrome de dor pós amputação, oncologia e Alzheimer. Os locais de aplicação podem ser: no local afetado, pontos gatilhos, terapia segmentar como as zonas head, terapia regional em gânglios e ao redor de nervos, terapia de infusão sistêmica e terapia de campo interferente (Weinschenk, 2012).

Segundo Bravo-Monsalvo et al. $(\underline{2008})$ a dose máxima do cloridrato de procaína a $0,7 \%$ quando utilizada de forma intradermal é de $7 \mathrm{mg} / \mathrm{kg}$, em um estudo em cães com dermatite atópica utilizou se também a dose endovenosa de $0,1 \mathrm{mg} / \mathrm{kg}$.

\section{Relato de caso}

Uma cadela da raça Yorkshire de quatro anos de idade foi atendida no Centro de Estudos em Clínica e Cirurgia em Animais (CECCA - PUC Minas), com a queixa de anestro persistente há cerca de dois anos. Em seu histórico, havia problemas de pele recorrentes, já tratados, uma cirurgia cesariana à qual foi submetida aos oito meses de idade, e uma aplicação de progestágeno, com o objetivo de impedir o cio, aos dois anos de idade. Após esta aplicação a cadela não ciclou mais. Ao exame físico, não foram encontradas alterações dignas de nota, a exceção da cicatriz decorrente da cesariana, a qual foi identificada como uma cicatriz tóxica e sendo um possível campo interferente. Foi realizada a aplicação de injeção intradérmica, subcutânea e intramuscular com seringa de $3 \mathrm{ml}$ e agulha de insulina na dose de $3 \mathrm{ml}$ de cloridrato de procaína a $0,7 \%$ na cicatriz em questão (Figura 1). Após dois meses deste tratamento, a cadela voltou a ciclar e entrou novamente no cio.

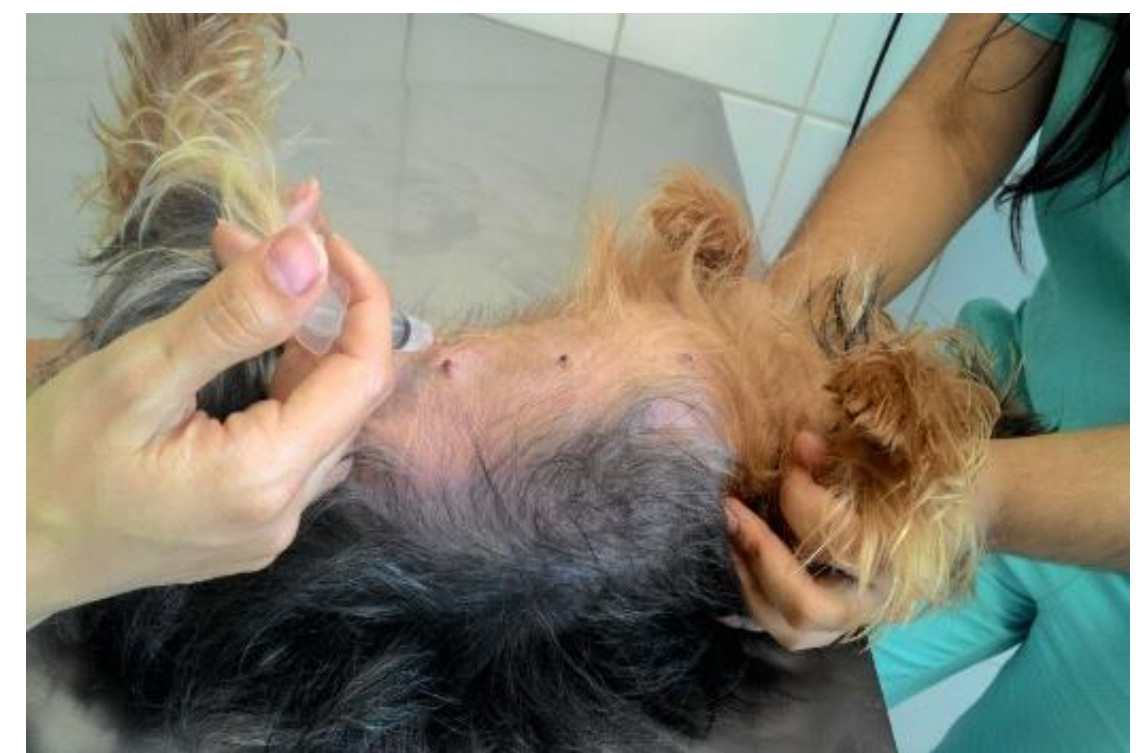

Figura 1. Aplicação de cloridrato de procaína a $0,7 \%$ intradérmica, subcutânea e intramuscular na cicatriz de cesariana. 


\section{Discussão}

Após o histórico de vida relatado pelo tutor identificou a cicatriz de cesariana como um campo de interferência e após o tratamento deste o animal restabeleceu o equilíbrio. Foi necessário somente uma sessão. O intervalo entre as sessões e a duração do tratamento é individual depende de cada caso.

Segundo Vall-Toscas et al. (2016) observa se êxito após aplicação intradérmica de procaína em cicatriz de cesariana como campo interferente em pacientes com relato de dor persistente pós parto, concluiu-se que independente do tempo de cirurgia o tratamento da cicatriz diminui a dor. Não necessariamente toda cicatriz cirúrgica ou acidental se comporta como campo interferente, isto vai depender de acordo com o histórico de vida do paciente e o possível bloqueio elétrico local ou a distância gerada pelo dano local.

Dentro da prática clínica de Terapia Neural observa se que corpo e mente estão ligados, deve se considerar episódios emocionais e não somente físicos no levantamento da história de vida do paciente. É um tratamento integral que observa o paciente como um todo e suas relações com o entorno (Navarro \& Pinilla, 2013). Segundo Speransky (1954) o sistema nervoso participa e coordena todos os acontecimentos do corpo. Um acometimento em determinado local reflete no corpo como um todo.

\section{Conclusão}

A Terapia Neural é um sistema médico, com uma concepção diferente da causa das enfermidades. Citando Hipócrates " "Não existem doenças, mas sim doentes". O objetivo da TN é que o corpo consiga após o tratamento realizar uma auto regulação corporal. Quando o paciente não responde ao tratamento pode estar com outros campos interferentes ou um bloqueio no sistema de auto regulação. Esta condição pode ser causada por certos medicamentos alopáticos como esteroides, antibióticos, psicofármacos, alimentos inadequados, condições emocionais e ambientais desfavoráveis e por intoxicações, por metal pesado por exemplo. No caso descrito uma única aplicação de cloridrato de procaína a $0,7 \%$ na cicatriz de cesariana foi o suficiente para que a cadela reencontra se seu equilíbrio e retornasse a ciclar normalmente.

\section{Referências}

Álvarez-Edwin, D. \& Andrés, P. A. C. 2017.Terapia Neural: los dientes y su relación com el organismo. Revista OACTIVA UC Cuenca. 2(1): 51-54.

Akcal, A.; Karsidag, S.; Yildiz, K.; Yesiloglu, N.; Akcal, M. A. \& Kabukcuoglu, F. 2015. The effects of locally applied procaine on wound healing. Archives of Clinical Experimental Surgery. 4: 41-45. Doi:10.5455/acess.20140606054447.

Bella, F. A. 2011. Cicatrices: Teoría de los campos interferentes. Revista Española de Podologia. XXII: 268-272.

Bravo-Monsalvo, A.; Vázquez-Chagoyán, J.; Gutiérrez, L. \& Sumano, H. 2018. Clinical efficacy of neural therapy for the treatment of atopic dermatitis in dogs. Acta Veterinaria Hungarica. 56:459469. doi: 10.1556/AVet.56.2008.4.4.

Cassuto, J.; Sinclair, R. \& Bonderovic, M. 2006. Anti-inflammatory properties of local anesthetics and their present and potential clinical implications. Acta Anaesthesiologica Scandinavica. 50:265-282. https://doi.org/10.1111/j.1399-6576.2006.00936.

Castro, R.A. 2011.Bases para la terapia neural em caninos y felinos. Buenos Aires: Dunken. 304p.

Cruz, Y. \& Fayad, R.N.2011. Microtúbulos y Terapia Neural: propuesta de uma investigación promisoria. Revista Med. 19: 82-92.

Egli, S.; Pfister, M.; Ludin, S.M.; De La Veja, K. P.; Busato, A.\& Fischer, L. 2015. Long-term results of therapeutic local anestesia (neural therapy) in 280 referred refractory chronic pain patients. BMC Complementary \& Alternative Medicine. 15:1-9. https://doi.org/10.1186/s12906-015-0735-z.

De La Torres Roses, M.M. \& Mendoza, R.L. 2008. La terapia neural: uma alternativa em el tratamiento de La litiasis renal. Revista Arch. Méd. Camagüey. 12: 1-9. 
Gonçalves, B. A. L., Vianna, L. R. \& Andrade, C., C. 2019. Alopecia Psicogênica em gato tratada com $\begin{array}{llllll}\text { Terapia Neural: } & \text { Relato de }\end{array}$ https://doi.org/10.31533/pubvet.v13n12a463.1-6

Gonçalves, B. A. L.; Vianna L. R.; Andrade, C.C. \& Oliveira, J. S. G. 2020. Anestésicos locais e a ação antimicrobiana, uma opção em microrganismos multirresistentes: Revisão de Literatura. Revista $V$ \& Z Minas. 145: 29-32.

Gonçalves, B.A.L; Vianna, L.R. \& Andrade, C.C. 2020b. Terapia Neural: uma opção de tratamento em pacientes oncológicos. Reviews Multidisciplinary. 3: e2020014. https://doi.org/10.29327/multi.2020014

Klinghardt, D.K. 2002. Neural therapy. Explore 11:01-05.

Muri, E.M.F.; Sposito, M.M.M \& Metsavaht, L. 2010. Efeitos secundários potencialmente desejáveis dos anestésicos locais. Acta Fisiatr.17(1): 28-33.

Navarro, K. \& Pinilla, L. 2013. Los aportes de Henry Head a las bases neuroanatómicas y fisiológicas de la de terapia segmento. Revista de los estudiantes de medicina de la universidade industrial de Santander. 26(3): 33-44.

Speransky, A. D. 1954. Bases para una nueva teoría de la medicina. Buenos Aires: Psique.

Vall-Toscas, C.; Sardá-Barea, E.M.; Martinez-Bueno, C. \& Zaragoza-Marfà, A. 2016. Dolor persistente posparto. Tratamiento con procaína subdérmica (terapia neural). Matronas profesión. 17 (4): 143-148.

Vianna, L.R.; Gonçalves, B.A.L. 2016. Nasce uma nova especialidade na Medicina Veterinaria Brasileira: Terapia Neural e Odontologia NeuroFocal. Revista V\&Z Minas. 130:16-21.

Vianna, L.R. \& Gonçalves, B.A.L. 2017. Entenda a terapia neural. Revista CFMV. 74, 44-47.

Vianna, L. R. \& Gonçalves, B. A. L. 2020. pH e condutividade do cloridrato de procaína em diferentes concentrações utilizadas na terapia neural. Multidisciplinary Science Journal. 3:e2021002:1-5. https://doi.org/10.29327/multiscience.2021002

Weinschenk, S. 2012. Neural therapy - a review of the therapeutic use of local anesthetics. Acupuncture and related therapies. 1: 5-9. https://doi.org/10.1016/j.arthe.2012.12.004.

Histórico do artigo:

Recebido: 8 de janeiro de 2021

Aprovado: 23 de fevereiro de 2021.
Licenciamento: Este artigo é publicado na modalidade Acesso Aberto sob a licença Creative Commons Atribuição 4.0 (CC-BY 4.0), a qual permite uso irrestrito, distribuição, reprodução em qualquer meio, desde que o autor e a fonte sejam devidamente creditados. 